\title{
Research on Energy-Saving Small Cell of LTE Network
}

\author{
Yalin Duan and Yongbin Xie
}

\begin{abstract}
With the vigorous development of the LTE and the demand for green communication, new energy-saving technologies and deployment solutions also become the research emphasis of the construction of green cell. Heterogeneous network is one of the popular topic of green communication, while the small cell is an important part of LTE heterogeneous network. This paper presents the type and application scenario in current small cell network, and the composition of heterogeneous networks. In the heterogeneous network composed by Macrocell and small cell, putting forward the CRE energy-saving scheme in hot spots of city area, as well as the energy-saving construction scheme of preventing fire, cutting and poaching.
\end{abstract}

Index Terms-LTE, heterogeneous network, small cell, energy-saving.

\section{INTRODUCTION}

With the rapid development of mobile communication technology and the continuous improvement of people's requirements of high bandwidth and speed, wireless communications, particularly wireless mobile communications has undergone several leap in a few years. From the first generation of analog communication system, the second generation of digital mobile communication system, the third generation of mobile broadband mathematics communication system to the fourth generation of mobile communication system. At the same time, environmental pollution and energy consumption of the problems of the communications industry is increasingly serious.

From the distribution of the energy consumption of the entire mobile network equipment, the base station equipment accounted for $95 \%$ of energy consumption [1]; from the consumption point of view, in 2013, China's annual consumption of the entire communications industry has more than 20 billion $\mathrm{kw}$, the mobile communication network consumption accounted for the majority. Among them, the total power consumption of the base station has accounted for $60 \%$ to $70 \%$ of the overall operation and maintenance of telecommunications operators total electricity consumption. According to statistics, in 2014, China Mobile, the construction of base stations is expected to 50-55 million units. China Unicom expects the construction of $4 \mathrm{G}$ base stations to be more than 50,000. China Telecom is expected to about 200,000 [2]. Increasing the number of base stations, the energy consumption is also growing. Therefore, how to reduce the energy consumption of the base station effectively,

Manuscript received May 20, 2015; revised November 4, 2015.

The authors are with the Xi'an University of Post and Telecommunication, Xi'an Shannxi, China (e-mail: 286063534@qq.com). to create green energy wireless network, a common concern of communication industry .

Now, with the LTE licenses issued, the major operators are conducting large-scale LTE network construction. LTE frequency bands is relatively high, the power loss is larger, construction costs is increasing, the traditional base-building is difficult to meet the requirements of the depth of coverage of complex urban environment and the largest broadband wireless data services. Therefore, in addition to considering the traditional wide-area network coverage, LTE also need to consider the ways of heterogeneous network capacity to absorb the depth of coverage and hotspot. For this reason, many researchers and equipment manufacturers to develop and present a variety of heterogeneous network coverage of technology, including various types of small base stations, due to small size, low power, flexible deployment and other advantages, not only improve the network blind coverage, also resolve shortage problem to build a network base station site, and also play an important role in providing high-bandwidth data services, a small cell, is expected to become an important means of the underlying LTE network coverage in the future.

\section{SMall CELl}

\section{A. Introduction of Small Cell}

The small cell generally refers to a low-power wireless access node, in authorized, unauthorized frequency bands, small size, low power consumption [3]. Small cell is mainly used the following three scenarios: Firstly, use a macrocell coverage area, mainly to expand the coverage of the network edge, improve network speed. Such small cell typically deployed in hot spots of the city. Secondly, using outside the coverage area of a macrocells of a macro cell to supplement the reach of the blind, such as the number of hot spots within the scope of the suburbs. Thirdly, is mainly used for indoor environment such as office space and home interior environment. There are several different smallcell types such as Microcell, Picocell, Femtocell and a relay station (Relay) and so on. Among them, the Microcell is mainly for outdoor fill-blind, and filling hot scene, with the comprehensive advantages of fast and flexible, cnost-effective; Picocell is mainly for indoor public places, with comprehensive advantages of a low cost, and ease of deployment; Femtocell is mainly for residential users, with easy installation, quick deployment and other advantages; Relay is mainly for no wired backhaul scenario conditions, equipped with the advantages of expanding cell coverage area and improving the system capacity, environment of having no return fibers and no equipment room, so that we can reduce the number of base stations and reduce establishment costs significantly. 
Table I shows a variety of different types of transmission power of the base station, the access object and coverage. Microcell and picocell which can be seen as the miniaturization of macrocells, in addition to the different transmit power, but their position in the network with the same macrocells, and the transmission and processing of information from and macrocells are the same. Femtocell is mainly used in network coverage poor residential area, there is a strong closed, users can purchase and install by themselves. Users can connect core network via broadband or heterogeneous gateway access. Relay is a relay node between macrocells and the user, mainly for messaging [4].

TABLE I: POWER ACCESS OBJECT, COVERAGE OF DIFFERENT BASE STATION

\begin{tabular}{|c|c|c|c|}
\hline type & Power $(\mathrm{dBm})$ & access object & coverage $(\mathrm{km})$ \\
\hline Macrocell & $38-46$ & All users & $1 \sim 30$ \\
\hline Microcell & $30-36$ & All users & $0.1 \sim 0.5$ \\
\hline Picocell & $20-30$ & All users & $0.01 \sim 0.1$ \\
\hline Femtocell & $10-20$ & Closed user & $0.01 \sim 0.12$ \\
\hline Relay & $23-30$ & All users & $0.1 \sim 1$ \\
\hline
\end{tabular}

\section{B. Heterogeneous Network}

Green Communications, refers to the ideas of energy conservation, reducing environmental pollution, reducing the waste of resources and decreasing the hazards to environmental and human for the next generation of communication, it mainly uses innovative and efficient power amplifier, multi-carrier, distributed network , intelligent temperature control and the use of new energy technologies to transform the base station and to achieve the purpose of reducing energy consumption, and ultimately to achieve harmony between human and nature , to achieve sustainable development. Therefore, the base station energy-saving technology is the key to energy-efficient of whole base stations of mobile communications network. At present, the governing energy-saving technology of base station are including slimming technology of base station, multi-carrier frequency and modular devices, that are the primary equipment energy-saving technologies; in addition to improving power amplifier efficiency for energy-saving, distributed base station technology, the base station sleep Energy-saving Algorithm technology [5], residential scaling technology, intelligent temperature control technology, self-organizing network technology, heterogeneous network technologies, new green energy technologies are included [6]. Among them, heterogeneous networks technologies is a hot pots of LTE base station deployment.

Heterogeneous networks is the use of various existing wireless communication systems, through coordination between different systems, working together, is to learn from each other between multiple systems, an effective means to meet future mobile communications business requirements, can be integrated to play their respective advantages. In the macrocells system, some hot spots, such as offices, shopping malls, restaurants, or other zones away from the edge area of a macrocells, adding a small base station, it will form a heterogeneous network of macro-small base stations. This LTE heterogeneous networks is a variety of different power station.

From Fig. 1, we can see that the other base stations can directly communication with the

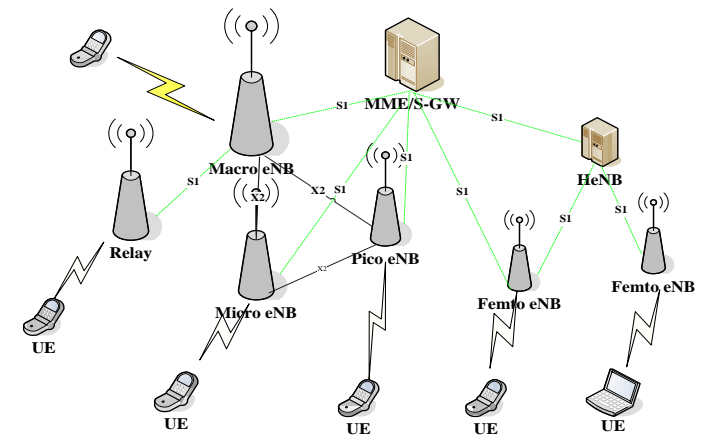

Fig. 1. LTE heterogeneous networks.

MME / S-GW (Core Network) through S1 (base station and the packet core network interface) .in addition to Relay, while Relay can only communicate with the Relay eNB. Macro eNB, Micro eNB, and Picoe NB also can communicate through $\mathrm{X} 2$ (base stations and base station interface). The Femto-eNB not only can communicate with the MME/ S-GW, but also communicate with HeNB (Home eNode B, femtocell access point), which is unique to the home base station gateway. Although LTE heterogeneous network more complex compared to other homogeneous networks, it can effectively disperses base pressure, shorten the distance between the base station and the user, it not only greatly improve the throughput capacity of the network, also improve the overall energy efficiency of the network [7].

\section{ENERGY-SAVING CONSTRUCTION SCHEME}

Deployment target of the small cell is mainly aimed at hot spots and blind zones, regardless of whether the areas have been macrocell coverage.

So, how to handle the relationship between the small cell and macrocell is crucial. As early as on the Mobile World Congress in Barcelona this year, Ericsson had proposed the concept of heterogeneous networks. Currently, the industry most proposed same heterogeneous network solution that networking with same frequency between the macrocell and the small cell, to correct the interference by macrocells with the collaboration of small cells. To some extent, in different scenarios, small cell network is the way of macrocell to extend and enhance the capacity, is not a simple overlay network.

Stereoisomeric LTE network schemes are divided into covering-layer and capacity-layer networking solutions. Covering layer mainly to meet the needs of wireless coverage, achieved by microcells which have to been qualified site construction conditionsand easy installation and maintenance. capacity layer mainly under the covering layer, uses the smallcells to provide basic network coverage. The hotspot areas of high traffic capacity demands, the places that difficult to deployment and the sites that difficult to coordinate use microcells, picocells, femtocells to install to increase the cell depth of coverage, increase network capacity and enhance the user perception [8]. According to three-dimensional network characteristic of heterogeneous networks, the paper chooses two two special scenarios that urban dense areas and remote sparse to discuss the networking technology of heterogeneous 
networks.

\section{A. Energy-Saving Programs of Densely Urbanized Areas}

Densely urbanized region refers to buildings, shops, crowded areas and the local areas of high demand for data services. LTE coverage of densely urbanized area is mainly possible increase network capacity, especially to improve the rate of edge users, improve the frequency utilization. three-dimensional network of this scene is based on the principle of hierarchical network covering physically. Generally, Macro eNB provide the basis for coverage, and Micro eNB, Pico eNB and Home eNB to cover blind spots and hot spots for supplement coverage, Relay[9] mainly extends coverage of Macro eNB, including streets, basements and other places.

Now, taking urban hot spots as an example, this paper puts forward macrocell-picocell energy-saving deployment scheme of heterogeneous network. In this type of heterogeneous network, the effect of picocell are mainly two things: for one thing, It is in the coverage area of a hotspot deployment of macrocell, providing services for users under a dense areas and to reduce the burden of macrocell; for another thing, to improve the quality of the communication on the edge of cell at the edge of the macrocell. According to the conventional coverage scheme, in hot spots, the user selects the base station which transmits a signal according to size of the base station signal SNR of base station. Then the users that picocell serves will be less than the macrocell, in other words, the area covered by the picocell is far more smaller than the area covered by the macrocell. This does not give full play to the efficacy of picocell. Only increasing slightly the proportion of area covered by the picocell in a whole residential areas, the effect of picocell can be slightly more better. That is keeping picocell transmit power unchanged, expanding picocell coverage area of CRE program.

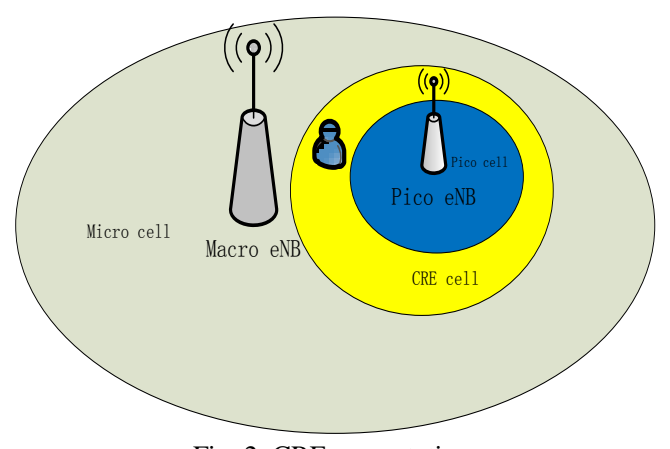

Fig. 2. CRE presentation.

The basic idea of CRE shown in Fig. 2, the selection criteria based on the reference information received power (Precv) cell, the macrocell coverage area as "Macro cell", i.e., the coverage area of the picocell as "Pico cell". When CRE mode is enabled, the coverage area of the picocell would expand to "CRE cell". CRE is the basic principle when the user selects a base station for communication, the picocell plus an offset (Poffset). For the macrocell, the offset is 0 , and for picocell, the offset is greater than 0 .

When the UE is under the coverage of a macrocell,

Precv of macrocell > Precv of picocell;

When the UE is under the coverage of a picocell,

Precv of picocell > Precv of macrocell;
When the UE is under the coverage of CRE region,

Precv of picocell + Poffset $>$ Precv of macrocell ;

Obviously, if a UE in a picocell CRE area, although the power received from macrocell are larger, receives the base station's power, it selects the picocell, which raises the number of UE of picocell serves, expanding the coverage of picocell.

Three-dimensional network of programs covering urban agglomeration areas theoretically solves "heating" issue of concentrated areas. The biggest problem is that there are still inter-cell interference coordination. This paper gives the following suggestions: Firstly, rational planning of the location of small base stations and coverage radius. Secondly, deployment of the picocell uses rationally of spatial separation of buildings, reducing inter-cell signal interference. Finally, interference coordination technology of soft frequency reuse is recommended. Heterogeneous networks soft frequency reuse is all the frequency resource is divided into several mutually orthogonal subbands. The edge of a macrocell use a part, the remaining resources can be used in the central region of the macro cell. The small base station within the coverage of Macrocell uses frequency resources and the macrocell orthogonal to the edge. Subcarrier of different macrocell edges orthogonal to each other, subcarriers of macrocell edge and subcarriers of picocell within the coverage of Macrocell orthogonal to each other, suppressing interference at the edge of cell effectively [10].

\section{B. Energy-Saving Programs of Remote Areas}

In remote areas, due to the sparse population, less business, complex terrain environment and high construction cost of the base station, operators also face the pressure of high investment and low return. To solve the problems of remote signal coverage, the macrocells of high power generally are used, which is a large investment, constrained by environmental conditions, high construction cost and operating costs. Therefore, actively studying to how to use green, environmentally friendly, low-cost means to solve the coverage problem in remote areas is of great strategic significance.

Taking remote areas, the forests an example, this paper puts forward macrocell-smallcell energy-saving deployment scheme of heterogen- eous network.In recent years, With the global warming, the increase of population and social demand, the forest is facing a growing threat: deforestation, forest fires, area shrinking, besides wild animals are hunted, biodiversity has been severely destructed. If we can install surveillance or alarm system in the forest, it can effectively prevent poaching and deforestation of forest fires. In June 2014, an American charitable company, proposed "recycling of used smartphones and protecting rainforest" proposal. They recovered the phones and making a unified transformation, and they used depth-customized version of Android operating system, power efficiency, resource management and audio were optimized specifically. The phone is fixed on the crown, use solar panels to supply electricity, use highly sensitive microphone to capture the sound of the surrounding area, including any frequency chainsaw sound of gunfire, animal whine and other sounds. Through a network of open source software, compressed sound files will transfer to the cloud 
server for analysis. In just a few seconds, the audio data is analyzed, and sending to remind local law enforcement through GSM [11]. Recycling old mobile phones to secondary exploitation, this proposal for protection of forest ecosystems is very valuable, but there are still many factors not taken into account, such as whether the device can be deployed simultaneously to achieve forest fire early warning, as well as to report reliable and timeliness information in the forest areas of relatively weak signals.

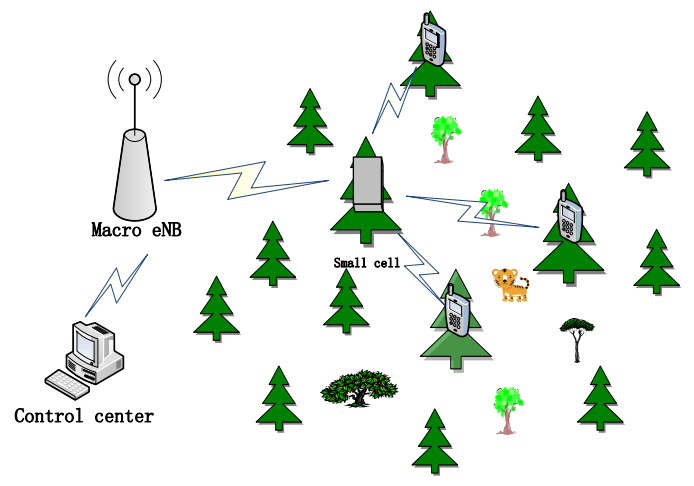

Fig. 3. Forest small cell deployments.

Taking all these factors, we consider the placed small green intelligent small cells on the basis of their proposal in the forest area, which forming a heterogeneous network with macrocell, as shown in Fig. 3. The so-called "green" refers to use the renewable energy sources (such as solar) as base station power, eliminating the expenditure for maintenance and replacement of the battery. The so-called "intelligent" refers to use intelligent temperature control [12], fireworks identification, fire point positioning and other technologies to implement forest monitoring, identification and location of the forest fire. We cover such small cells in the forest areas, on the one hand to provide good coverage to phones to capture audio, preventing deforestation and poaching. On the other hand, the small cells use solar panel, self-sufficiency, greatly reducing maintenance costs, and intelligent phones can real-time monitoring forest conditions, reducing the incidence of forest fires to a minimum. As long as small cells and intelligent phones deployed rationally according to the terrain and environment, the security of the entire forest areas can be guaranteed.

\section{CONCLUSION}

With the advancement of wireless communications research, different types of small base stations will be more widely used in wireless communications networks. And its roles played in $4 \mathrm{G}$ network construction has been widely recognized by the industry [13]. In the future, During the LTE network planning, a multi-level, hybrid network coverage pattern will be adopted.

Through the base stations of different types, different mutual integration techniques, the users will be provided high-speed and high-quality mobile communication network. At the same time, energy-saving resources bring new life to the Green Communications. Although now there have a collection of energy sources technologies and equipments to collect green energy sources, theirs application in wireless communication is not widespread, especially in the aspects of protection of forests and wildlife. So green and intelligent technology of small cell require further study as a hot topic in the current green communication fields.

\section{ACKNOWLEDGMENT}

This paper is sponsored by National High Technology Research and Development Program of China (863 Program) (GN: 2014AA01A703, 2014AA01A705).The authors would like to thank anonymous referees for their very constructive comments and corrections of errors.

\section{REFERENCES}

[1] T. Han and N. Ansari, "On optimizing green energy utilization for cellular networks with hybrid energy supplies," IEEE Transactions on Wireless Communications, vol. 12, no. 8, pp. 3872-3882, 2013.

[2] Green Communication. [Online]. Available: http: //www.cnii.com.cn/20080623/ca601066.htm. 2009

[3] X. S. Su, "Reasearch on the lte small cell construction strategy," Telecommunication Bulletin, no. 10, pp. 13-16, 2014.

[4] Y. X. Meng, "Study the techniques on energy saving of mobile communication base station," Shanghai Jiaotong University, 2012.

[5] E. Oh, K. Son, and B. Krishnamachari, "Dynamic base station switching-on/off strategies for green cellular networks," IEEE Transactions on Wireless Communications, vol. 12, no. 5, pp. 2126-2136, 2013.

[6] D. Q. Feng, C. Z. Jiang, and G. B. Lim, "A survey of energy-efficient wireless communications," pp. 1-12, 2013.

[7] Y. F. Lou, "Research on energy-efficient of picocell in heterogeneous network of LTE," University of Electronic Science and Technology, pp. 36-38, June 2013.

[8] Nokia Siemens Networks and Nokia, "Discussion of pico node range extension benefits," 3GPP TSG RAN WG1 \#62-bit Meeting, Xi'an, China, 2010

[9] S. P. Li, G. D. Zhang, and F. S. Liu, "Application research of LTE small cell," Data Communication, pp. 9-11, April 2014.

[10] The Small Base Station Become Protagonist of LTE Heterogeneous Networks Covering Scene. (January 2013). [Online]. Available: http://www.enet.com.cn

[11] New Use for old mobile phones. (June 2014). [Online]. Available: http://www.uuhomepage. com/staticpages/20140626004429.html

[12] S. H. Liu, "Study and its application of base station energy efficiency intelligent management in mobile communication network," Shanghai Jiao Tong University, June 2012, pp. 5-7.

[13] F. Qin and Q. L. Yu, "4G era - small base move towards the big stage," Shanghai Information Technology, pp. 24-26.

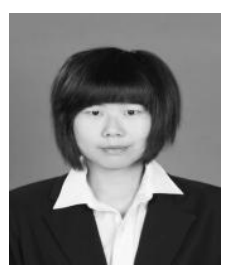

Yalin Duan was born in May 1990. She graduated in Xi'an University of Post and Telecommunication, Xi'an Shannxi, China. Her major is communication and information system. Her main research direction is broadband wireless communication technology. Now, she is as a internship in Datang R\&D Center, mainly engaged in the development of LTE terminal.

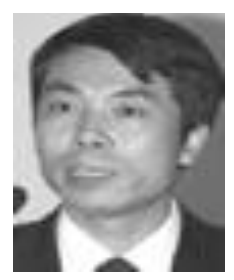

Yongbin Xie is a professor, doctor, tutor in Xi'an Shannxi, China. He engaged in TD-SCDMA, TD-LTE research, since 2000 . He is mainly engaged in research and development of third generation mobile communication technology. He has organized a system-wide WCDMA, TD-SCDMA access R \& D network and terminals. He was invited to $\mathrm{Xi}$ 'an University of Posts and Telecommunications in 2011, and was named distinguished professor of Shaanxi Province "Hundred Talents Program". Nearly 30 kinds of papers have published, and have seven by EI. 